\title{
DETECTION OF THE CTG REPEAT EXPANSION IN CONGENITAL MYOTONIC DYSTROPHY
}

\author{
Kazuhiro OHya, ${ }^{1, *}$ Nobutada TACHI, ${ }^{2}$ Toshiya SATO, ${ }^{1}$ Shinichiro Kon, ${ }^{3}$ \\ Kokichi KIKUCHI, ${ }^{3}$ and Shunzo CHIBA ${ }^{1}$ \\ ${ }^{1}$ Department of Pediatrics and ${ }^{3}$ First Department of Pathology, \\ Sapporo Medical University School of Medicine, South 1 West 16, \\ Chuo-ku, Sapporo 060, Japan \\ 2 Sapporo Medical University School of Health Science, \\ South 1 West 16, Chuo-ku, Sapporo 060, Japan
}

Summary Myotonic dystrophy (DM) is caused by an abnormal expansion of an unstable CTG trinucleotide repeat in the $3^{\prime}$ untranslated region of mRNA encoding a putative serine/threonine protein kinase. We analyzed 59 patients with DM (28 congenital DM families: 27 families with maternal transmission and 1 paternal transmission) and 27 normal control subjects to evaluate their CTG repeat size between DM patients and the normal controls, and to search for a correlation between the clinical characteristics of congenital DM (CDM) and CTG repeat expansions. Analysis was on the basis of the Southern blot and polymerase chain reaction (PCR) methods, and by direct sequencing of PCR amplified CTG repeats. Analysis of intergenerational differences in the CTG repeat size for mother-child pairs showed a positive correlation $\left(y=1.0384 x+1265.2, r^{2}=0.311\right)$. In addition to the strong parental bias, this group showed genetic anticipation. There was a significant correlation of the CTG repeat expansion with disease severity. The largest CTG repeat expansion (2,293 CTG repeats) on average belonged to the severe CDM group, and the smallest (129 CTG repeats) to the subclinical DM group. The mutant allele of an asymptomatic father in the paternally transmitted pedigree revealed $75 \mathrm{CTG}$ repeats, demonstrating that he was a DM protomutation carrier.

Key Words myotonic dystrophy (DM), congenital form, trinucleotide (CTG) repeat mutation, parental bias, genetic anticipation

\section{INTRODUCTION}

Myotonic dystrophy (DM) is an autosomal dominant, multisystemic disorder

Received April 22, 1996; Revised version accepted November 14, 1996.

* To whom correspondence should be addressed: Department of Pediatrics, Asahikawa Red Cross Hospital, 1-1 Akebono, Asahikawa 070, Japan. 
characterized by myotonia, progressive muscle weakness and atrophy, cardiac conduction disturbances, cataracts, and abnormal glucose intolerance (Harper, 1989). DM is the most common form of adult muscular dystrophy, with an estimated incidence of 1 in 8,000 . Clinical features of DM are markedly variable, ranging from neonatal mortality (congenital DM, CDM) to a complete absence of symptoms. The age at onset of the disease and severity of the symptoms show extreme variations even among family members. CDM is associated with severe hypotonia, feeding difficulty, neonatal respiratory distress, developmental motor delay and mental retardation, distinct from the adult DM. Most children with CDM are the offspring of affected mothers (Höweler et al., 1989). The phenomenon of genetic anticipation, earlier age of onset and increasing severity of the disease in successive generations, always occurs in DM pedigrees (Ashizawa et al., 1993).

The gene responsible for DM (DM kinase gene) has been mapped to human chromosome 19q13.3. The complete sequence of the DM kinase gene is within a $14-\mathrm{kb}$ genomic region containing five contiguous $B a m \mathrm{HI}$ fragments and constituting of 15 exons.(Mahadevan et al., 1993a). The full length of mRNA of the DM kinase gene is estimated to be approximately $3.4 \mathrm{~kb}$ and encodes a $69-\mathrm{kDa}$ translational product of 629 amino acids which has no homology to any known protein sequences. The molecular basis of DM mutation is an unstable trinucleotide (CTG) repeat, located at the $3^{\prime}$ end of a transcript encoding a putative serine/threonine protein kinase (Brook et al., 1992; Buxton et alo, 1992; Mahadevan et al., 1992; Fu et al., 1992). The number of expansions varies markedly, even in a normal population from 5 to 30 , but in DM patients it ranges from 50 to several thousand copies (Tsilfidis et al., 1992). In asymptomatic individuals at a premutation stage, CTG repeat varies from 35 to 50 .

We analyzed $28 \mathrm{CDM}$ families and 27 normal control subjects to evaluate the CTG repeat size between DM patients and normal controls, and to clarify correlations between the clinical characteristics of CDM and CTG repeat expansions.

\section{MATERIALS AND METHODS}

Patients and blood samples. Blood samples were obtained from 59 DM patients from 28 families ( 27 families with maternal transmission and 1 paternal transmission; 16 severe CDM patients, $16 \mathrm{CDM}$ patients, 23 adult DM patients, and 4 subclinical DM patients; 25 mother-child pairs and 2 father-child pairs) and 27 normal control subjects. Peripheral blood leukocytes were taken from the patients and the normal controls. CDM was diagnosed based on clinical features and muscle pathology. We classified the patients into the following four groups on the basis of clinical manifestations and age of onset. (1) Severe CDM: these patients were characterized by clinical features such as the presence of polyhydramnios and reduced fetal movements, marked hypotonia with facial weakness at 
birth, neonatal respiratory distress, and feeding difficulty during the neonatal period. (2) CDM: they had facial weakness, generalized hypotonia at neonatal period, delayed motor development, and mental retardation. (3) Adult DM: they represented myotonia beginning at adult life, progressive muscle weakness and atrophy. (4) Subclinical DM: they were asymptomatic DM carriers with only cataracts.

Southern blot analysis. Genomic DNAs were prepared from peripheral blood leukocytes by the standard procedures (Sambrook et al., 1989). Seven micrograms of genomic DNA was digested with appropriate restriction endonucleases (EcoRI, BamHI, SacI, and BglI), separated by electrophoresis on $0.8 \%$ agarose gel, and transferred onto nylon membranes using a vacuum transfer apparatus (VacuBlot ${ }^{\mathrm{TM}}$ Transfer System, American Bionetics, USA). After prehybridization, the digested DNA was hybridized to radiolabeled p5B1.4 for $24 \mathrm{hr}$. The filters were washed in a stringency of $0.1 \times$ SSC containing $0.1 \%$ SDS and autoradiography was performed at $-80^{\circ} \mathrm{C}$ for 2 to 5 days. The probe $\mathrm{p} 5 \mathrm{~B} 1.4$ (Shelbourne et al., 1992), supplied by Dr. Keith Johnson, is a 1.4-kb BamHI fragment from cDNA25 (Buxton et al., 1992) subcloned into pBluescript SK+. This probe was labeled by random priming.

Polymerase chain reaction ( $P C R)$ amplification. PCR was performed using CTG region-flanking primers (Brook et al., 1992; Mahadevan et al., 1992). Cycling conditions were as follows: initial denaturation at $95^{\circ} \mathrm{C}$ for $3 \mathrm{~min}, 35$ cycles at $95^{\circ} \mathrm{C}$ for $1.5 \mathrm{~min}, 65^{\circ} \mathrm{C}$ for $1 \mathrm{~min}$ and at $72^{\circ} \mathrm{C}$ for $2 \mathrm{~min}$, followed by a final stage at $72^{\circ} \mathrm{C}$ for $7 \mathrm{~min}$. PCR products were electrophoresed on $2 \%$ agarose, blotted onto a nylon membrane, and probed with a $\left[\gamma^{32} \mathrm{P}\right] \mathrm{ATP}$ endlabeled $(\mathrm{CTG})_{10}$ oligonucleotides. Membrane was washed in $6 \times$ SSC containing $0.1 \%$ SDS and exposed to Kodak $\mathrm{X}$-ray film. CTG closely flanking primers were as follows:

primer \#101 (26 mer): 5'-CTTCCCAGGCCTGCAGTTTGCCCATC-3'

primer \#102 (27 mer): 5'-GAACGGGGCTCGAAGGGTCCTTGTAGC-3'

primer \#406 (22 mer): 5'-GAAGGGTCCTTGTAGCCGGGAA-3'

primer \#409 (20 mer): 5'-GGAGGATGGAACACGGACGG-3'.

Direct sequencing of $P C R$ product. DNA fragments containing the CTG trinucleotide repeats in leukocytes were amplified by PCR using the CTG repeat closely flanking primers, purified by electrophoresis through $2 \%$ agarose gel. Primer $\# 409$ was biotinylated at the $5^{\prime}$ end. Biotinylated single stranded DNA was prepared by Dynabeads ${ }^{\mathrm{TM}}$ M-280 Streptavidin (Dynal ${ }^{\circledR}$, Norway) procedures and sequencing was performed according to Sanger's dideoxy chain termination method using a Sequenase kit Ver.2 ${ }^{\mathrm{TM}}$ (U.S.B., USA) and ${ }^{35} \mathrm{~S}-\mathrm{d} A T P$. Nucleotide sequences were obtained by $6 \%$ polyacrylamide electrophoresis followed by drying and exposed to Kodak X-ray film for $24 \mathrm{hr}$. 


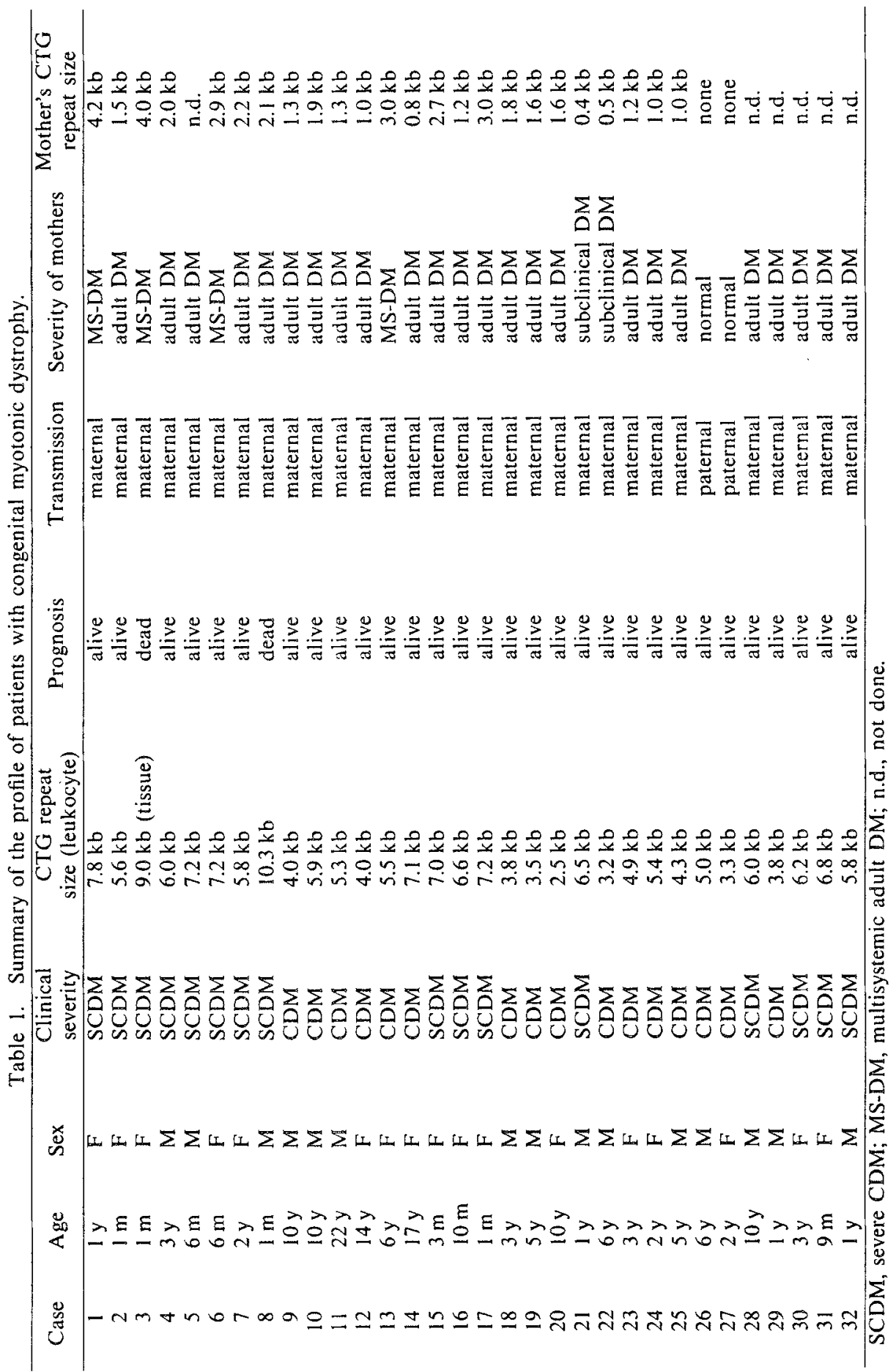




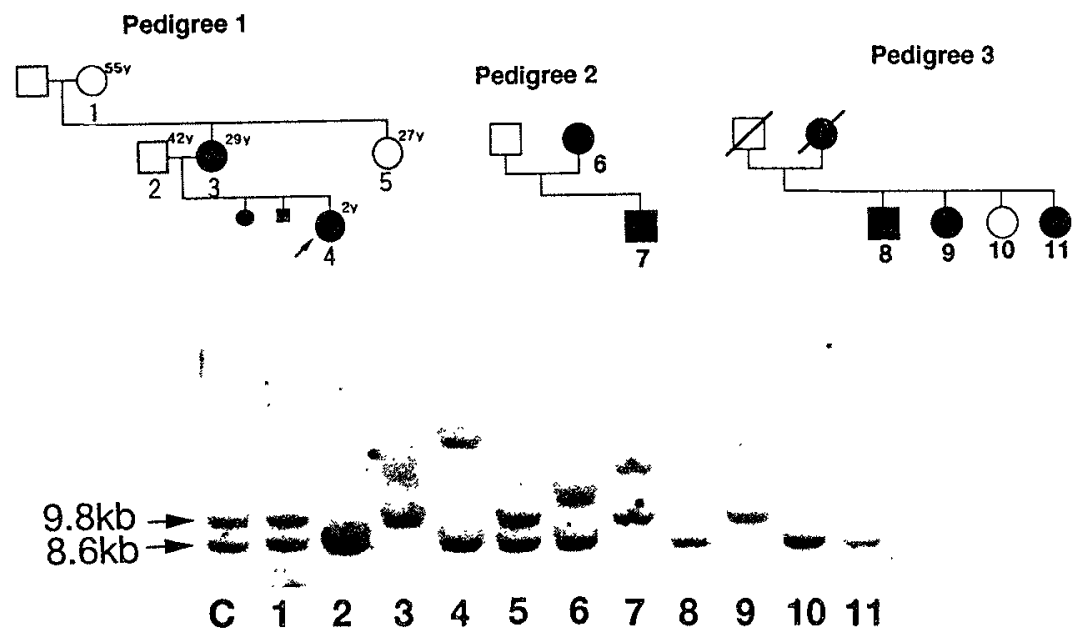

Fig. 1. Southern blot analysis of EcoRI fragments in three families with CDM. EcoRI-digested DNAs probed with p5Bl.4 showed an EcoRI polymorphism with 9.8 and $8.6 \mathrm{~kb}$ alleles in a normal population. In pedigree 1 , both an asymptomatic maternal grandmother (Subject 1) and a mother's unmarried younger sister (Subject 5 ) had 8.6 and $9.8 \mathrm{~kb}$ heterozygous alleles, and a father (Subject 2) had $8.6 \mathrm{~kb}$ homozygous alleles. They had no CTG repeat expansions. Mother (Subject 3) had adult DM; she had $9.8 \mathrm{~kb}$ and expanded smearing $14.2 \mathrm{~kb}$ alleles (about 1,400 CTG repeats). A girl with typical severe CDM (Subject 4) had $9.8 \mathrm{~kb}$ and expanded discrete $17.8 \mathrm{~kb}$ alleles (about 2,800 CTG repeats). In another two CDM families (pedigrees 2 and 3), adult DM patients had normal alleles and expanded alleles, and CDM patients had normal alleles and larger expanded repeat than adult DM patients. Squares, men; circles, women. Solid symbols indicate affected family members.

\section{RESULTS}

Table 1 summarizes the characteristics and results of CTG repeat expansions of the CDM patients. All the DM patients had variable CTG repeat expansions, and normal controls had no expansions. Figures 1, 2 (Southern blot analysis) and 3 (PCR analysis) were typical patterns of CTG repeat expansions in CDM pedigrees.

\section{Intergenerational differences in $C T G$ repeat size of mother-child pairs}

Analysis of the intergenerational differences in CTG repeat size for motherchild pairs (Fig. 4) showed a positive correlation $\left(y=1.0384 x+1265.2, r^{2}=0.311\right.$, $\mathrm{n}=25$ ). The present group of CDM showed a strong parental bias; all 27 pedigrees except one showed transmission from the affected mother. They showed genetic anticipation, i.e., earlier age of onset and increasing disease severity in successive 

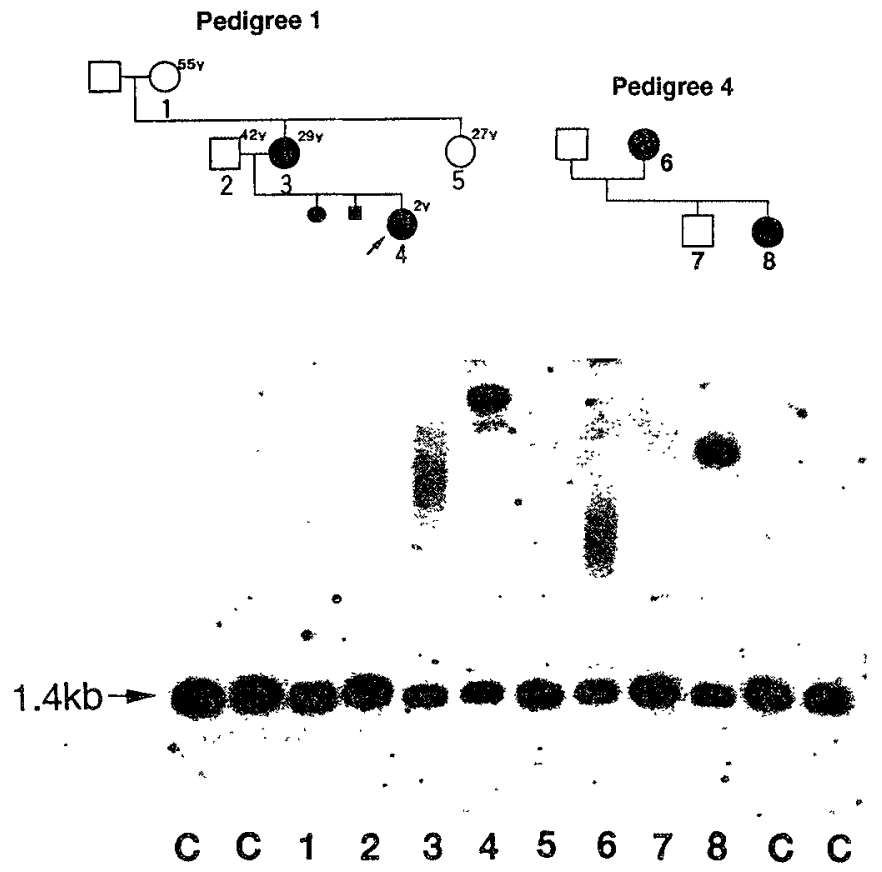

Fig. 2. Southern blot analysis of BamHI fragments in two families of CDM. BamHIdigested fragments can detect smaller allelic expansions of about 200 bp (65-70 CTG repeats). Patients in whom allelic expansion is ambiguous can be diagnosed. Southern blot analysis showed a single band of $1.4 \mathrm{~kb}$ in normal individuals. In pedigree 1, expanded CTG repeats were detected as smears in the mother with adult form of DM (Subject 3). A girl with severe CDM (Subject 4) had a normal band of $1.4 \mathrm{~kb}$ plus a larger discrete expanding band of $9.2 \mathrm{~kb}$. No expansion was detected in an asymptomatic grandmother (Subject 1), father (Subject 2), and an aunt (Subject 5). In pedigree 4, the expanded CTG repeat was detected as a smear in a mother with adult form of DM (Subject 6). A girl with CDM (Subject 8) had a normal band of $1.4 \mathrm{~kb}$ plus a larger discrete expanding band. No expansion was detected in an asymptomatic elder brother (Subject 7).

generations.

\section{Paternal transmission}

We previously reported the first CDM patients in a family in whom the transmission was paternal and showed the anticipation in both phenotype and genotype (Ohya et al., 1994). The father had a minimal expanding band of $1.6-\mathrm{kb}$ in BamHI-digested DNAs (data not shown). In determining the accurate number of CTG repeat of minimal expanded mutant alleles, the PCR using the CTG repeat flanking primers and direct sequencing of PCR products were performed. The mutant allele of the father revealed 75 CTG repeats (Fig. 5). 

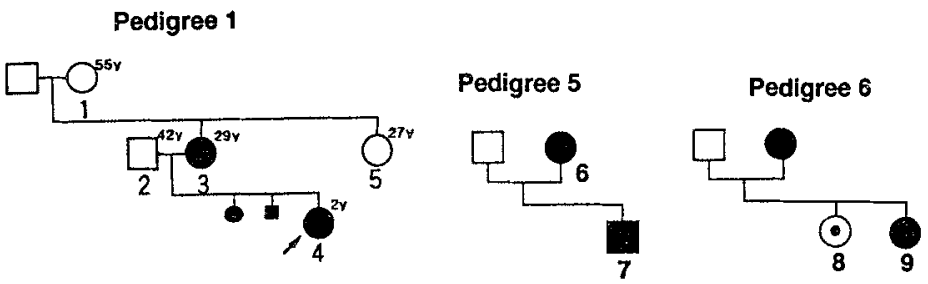

a

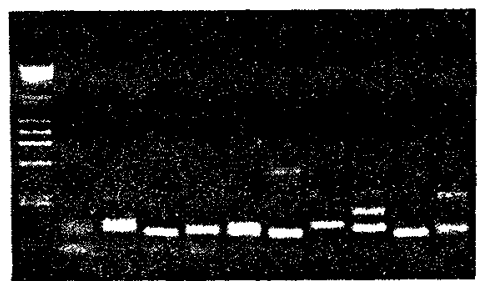

b
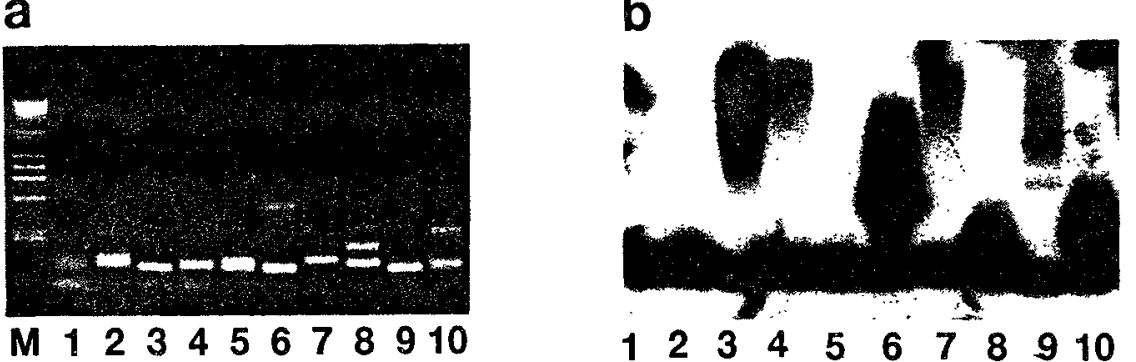

Fig. 3a: Ethidium bromide-stained 2\% agarose gel banding pattern of PCR-amplified CTG repeats region in congenital DM pedigrees. Ethidium bromide staining of the PCR-amplified CTG repeat region (using flanking primers \#101 and \#102) indicated that a grandmother (Subject 1) and an aunt (Subject 5) had two normal alleles of 125 base pairs (bp) and $149 \mathrm{bp} \mathrm{(5} \mathrm{and} 13$ CTG repeats). A father (Subject 2) had two normal alleles of $134 \mathrm{bp}$ and $155 \mathrm{bp}$ ( 8 and 15 CTG repeats). PCR-amplified DNA in a mother (Subject 3) and a girl (Subject 4) showed only the normal alleles of $125 \mathrm{bp}$ and $134 \mathrm{bp}$, respectively.

b. In Southern blot analysis probed with $5^{\prime}$ end radiolabelled (CTG) $)_{10}$ oligonucleotides, PCR products from the asymptomatic grandmother (Subject 1) and the aunt (Subject 5) showed a hybridized signal only above the normal alleles, while PCR-amplified DNA from the mother with adult DM (Subject 3) showed two distinct hybridized signals above both a normal allele and a mutant allele. The girl (Subject 4) had both a normal allele signal and a faint smearing of the hybridization signal above an expanded mutant allele. On the basis of the clinical features presented, the results of Southern blot analyses, and PCR amplification analyses, the girl (Subject 4) in pedigree 1 was confirmed as having CDM. The asymptomatic grandmother (Subject 1 ) and the aunt (Subject 5) were confirmed to be normal. In another two CDM families (pedigrees 5 and 6), adult DM patient (Subject 6) had normal alleles and expanded alleles, and CDM patients (Subjects 7 and 9) had normal alleles and larger expanded repeat than adult DM patients on Southern blots. Subject 8 (pedigree 6) was a subclinical DM. Size-standard DNA (M) is $\lambda$ DNA digested with Hindlll plus PhiX174 DNA digested with Hinfl The numbers of the lane 1 to 5 are the same ones as shown in Figs. 1 and 2 . Lane 10 showed a father of paternally transmitted CDM pedigree.

\section{Correlation between the number of CTG repeat and the age of onset}

There were significant correlations between the number of CTG repeats and 


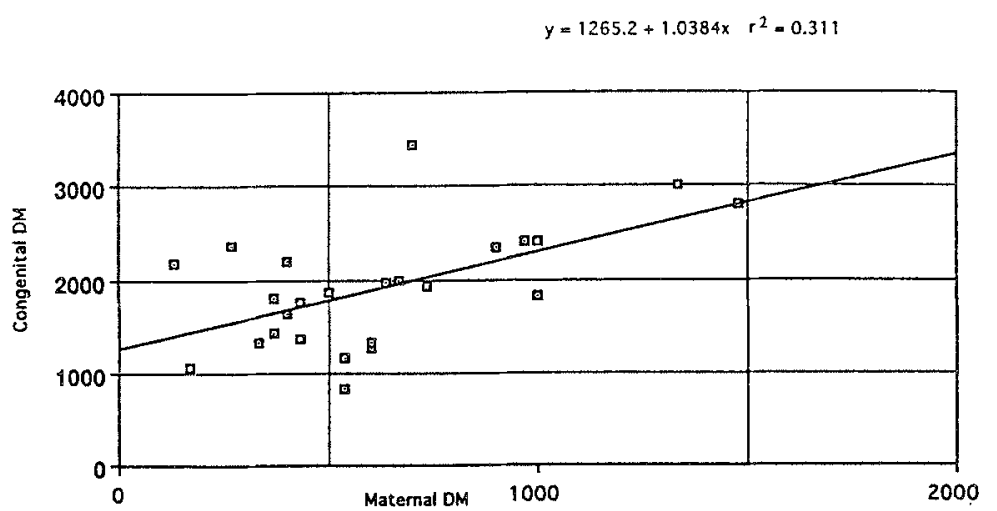

Fig. 4. Intergenerational differences in CTG repeat size of mother-child pairs. Analysis shows a positive correlation (see text). Almost all cases of CDM show parental bias, which is transmitted from affected mother, except one pedigree.

both the age of onset and disease severity (Fig. 6). The largest CTG repeat expansion on average belonged to the severe CDM group, and the smallest to the subclinical DM group. The severe CDM patients showed 2,293 4404 CTG repeats (mean $\pm S D$ ), CDM patients $1,445 \pm 400$ repeats, adult DM $581 \pm 234$ repeats, and subclinical DM showed $129 \pm 98$ repeats. There was a significant difference in repeat size among the four groups: $p<0.001$.

\section{DISCUSSION}

DM in our series showed quite variable phenotypes, ranging from CDM to a complete absence of symptoms. Genetic anticipation was observed in all the present pedigrees. The severity of CDM confirmed to correspond to the length of CTG repeats in mutant genes from peripheral blood leukocytes in our study. Mahadevan et al. (1992) suggested that a blurred or smeared appearance of expanded alleles seen on Southern blots in lymphocytes indicated somatic cell heterogeneity in the size of the expanded alleles. We also observed smeared expanded bands in several DM patients on Southern blots. In PCR analysis, we detected smeared bands of mutant alleles in all DM patients analyzed. Our molecular data in CDM were consistent with the results of previous reports (Tsilfidis et al., 1992; Mahadevan et al., 1992; Harley et al., 1992). Yamagata et al. (1994) reported characteristic relationships between CTG repeat mutations and clinical phenotypes in a Japanese population. Our study made the correlations clear between the triplet expansion and CDM clinical features.

The transmission of CDM was previously considered exclusively maternal. However, less than $10 \%$ of affected mothers gave birth to CDM infants. The risk of congenitally affected babies inherited from multisystemic DM mothers is 


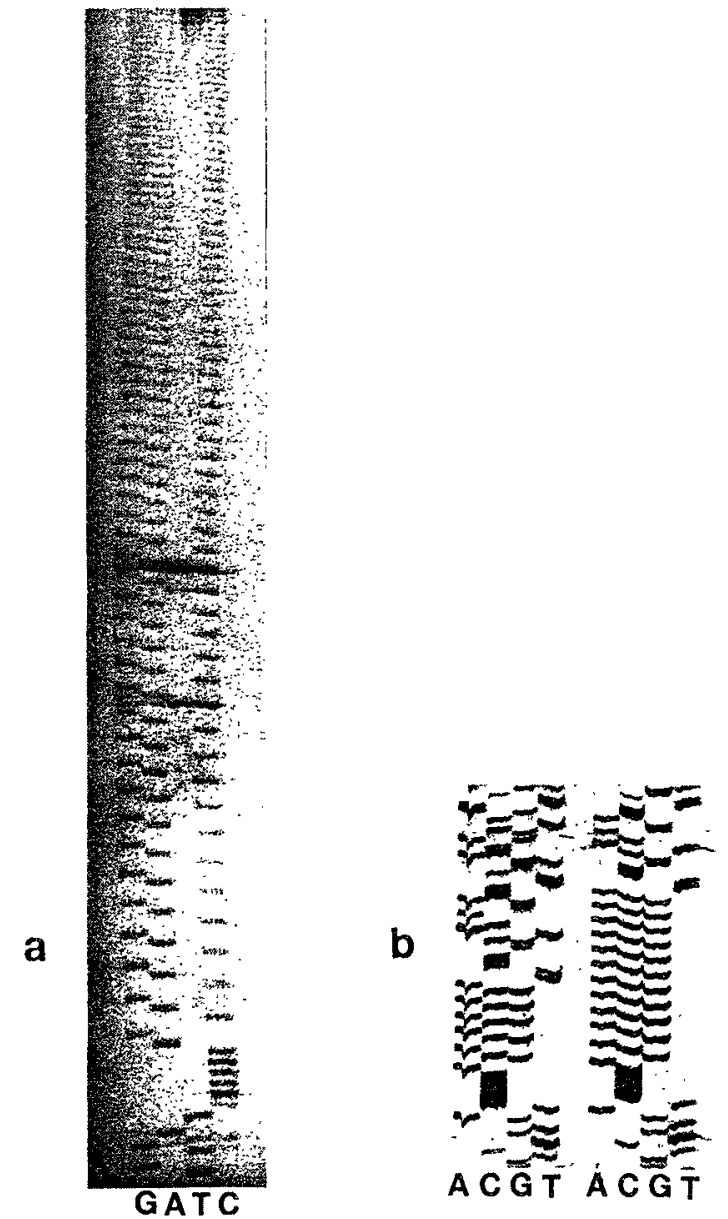

Fig. 5. Direct sequencing of a mutant allele of a DM premutation carrier in the paternally transmitted family pedigree and normal alleles, showing 75 copies of the CTG repeats (a) and 5 and 11 copies of normal alleles (b).

approximately $80 \%$ (Koch et al., 1991). Although we observed four such DM mothers and their infants with severe or lethal congenitally affected DM babies, the mothers had no understandings of high risk for bearing CDM offsprings. The CTG repeat expansion in DM mutations enables us to explain the genetic anticipation and phenotypic variability in DM, and to diagnose, patients who were asymptomatic or showed few of the classical signs. The identification of the DM mutation and the ability to perform a direct DNA analysis will enable physicians to provide families more accurate risk estimates and information for genetic counseling. Moreover, a DNA analysis will be especially helpful for prenatal diagnosis.

Vol. 42. No. 1, 1997 


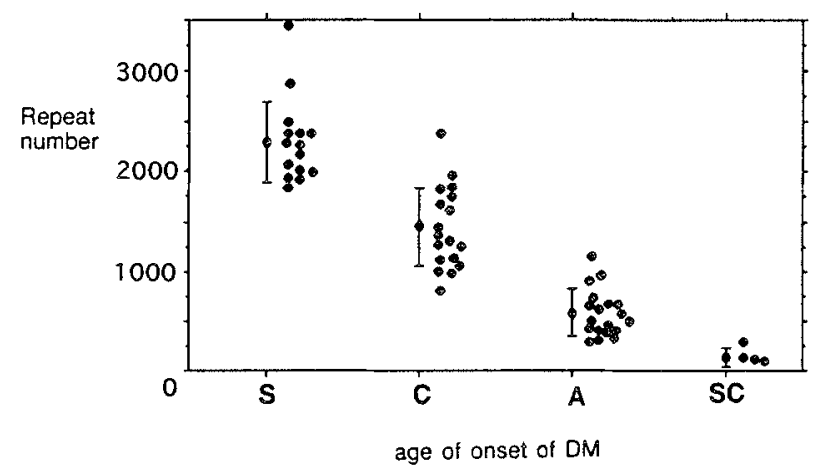

Fig. 6. Correlation between the number of CTG repeats and the age of onset of DM. There is a significant correlation of CTG repeat sizes with disease severity. The largest CTG repeat expansion on average belongs to CDM group, and the smallest to subclinical DM group.

We previously presented the first CDM case via paternal transmission, using DNA analysis (Ohya et al, 1994). Some DM patients have a very mild clinical signs associated with minimal amplification of the CTG repeat, ranging from 50 to 80 repeats. This class of DM mutation was called DM protomutation (Barceló et al., 1993). In our study, a mutant allele of the father reveals 75 CTG repeats. The asymptomatic father was a. DM protomutation carrier. We speculate that the changes in CTG repeat numbers may occur during oogenesis and spermatogenesis. Analysis of CTG repeats in this father's sperm may shed some light on paternal transmission of DM mutations.

Direct mechanism of the CTG repeat expansion in the DM gene is still unknown. But, an association of insertion and deletion polymorphisms located at intron 8 of the DM gene and the CTG repeat size was observed in Caucasian and Japanese populations (Yamagata et al., 1992; Mahadevan et al., 1993b). In DM patients, the expanded CTG repeat is associated with a $1-\mathrm{kb}$ insertion. The $(\mathrm{CTG})_{11-13}$ repeat was almost always associated with a deletion and the $(\mathrm{CTG})_{5}$, $(\mathrm{CTG})_{10}$, and $(\mathrm{CTG})_{19-30}$ repeats were associated with another insertion. The $(\mathrm{CTG})_{11-18}$ allele was stable and had no increasing change of repeats in successive generations, but $(\mathrm{CTG})_{5},(\mathrm{CTG})_{19-30}$ repeat alleles were quite unstable, almost always expanding in successive generations (Imbert et al., 1993). South African Negroids had a $(\mathrm{CTG})_{\mathrm{n}}$ allelic distribution that was significantly different from those in Caucasian and Japanese populations: the $(C T G)_{>19}$ repeat was very rare. These findings explained the absence of DM patients in South Africa (Goldman et al. 1994). Recently, Wong et al. (1995) reported that the size of expanded CTG repeat from the same adult DM patients showed a clear tendency to increase over 2- to 5-year intervals, suggesting an association of progressive shift to larger expansion size with advancing age (postnatal instability of CTG repeats). There- 
fore, our results require the verification of the CTG repeat expansion by prospective study with the same subjects after several years.

Acknowledgments We are grateful to Dr. Keith Johnson (Department of Anatomy, Charing Cross and Westminster Medical School, UK) for providing the probe p5B1.4.

\section{REFERENCES}

Ashizawa T, Dubel JR, Dunne PW, Dunne CJ, Fu Y-H, Pizzuti A, Caskey CT, Boerwinkle E, Perryman MB, Epstein HF, Hejtmancik JF (1993): Anticipation in myotonic dystrophy. II. Complex relationships between clinical findings and structure of the GCT repeat. Neurology 42: $1877-1883$

Barceló JM, Mahadevan MS, Tsifidis C, Mackenzie AE, Korneluk RG (1993): Intergenerational stability of the myotonic dystrophy protomutation. Hum Mol Genet 2: 705-709

Brook JD, McCurrach ME, Harley HG, Buckler AJ, Church D, Aburatani H, Hunter K, Stanton VP, Thirion J-P, Hudson T, Sohn R, Zemelman B, Snell RG, Rundle SA, Crow S, Davies J, Shelbourne P, Buxton J, Jones C, Juvonen V, Johnson K, Harper PS, Shaw DJ, Housman DE (1992): Molecular basis of myotonic dystrophy: expansion of a trinucleotide (CTG) repeat at the $3^{\prime}$ end of a transcript encoding a protein kinase family member. Cell 68: 799808

Buxton J, Shelbourne P, Davies J, Jones C, Van Tongeren T, Aslanidis C, de Jong P, Jansen G, Anvret M, Riley B, Williamson R, Johnson K (1992): Detection of an unstable fragment of DNA specific to individuals with myotonic dystrophy. Nature 355: 547-548

Fu Y-H, Pizzuti A, Fenwick RG Jr, King J, Rajnarayan S, Dunne PW, Dubel J, Nasser GA, Ashizawa T, de Jong P, Wieringa B, Korneluk R, Perryman MB, Epstein HF, Caskey CT (1992): An unstable triplet repeat in a gene related to myotonic muscular dystrophy. Science 255: $1256-1258$

Goldman A, Ramsay M, Jenkins T (1994) Absence of myotonic dystrophy in southern African Negroids is associated with a significantly lower number of CTG trinucleotide repeats. J Med Genet 31: 37-40

Harley HG, Rundle SA, Reardon W, Myring J, Crow S, Brook JD, Harper PS, Shaw DJ (1992): Unstable DNA sequence in myotonic dystrophy. Lancet 339: 1125-1128

Harper PS (1989): Myotonic dystrophy. 2nd ed. WB Saunders, Philadelphia, PA

Höweler CJ, Busch HFM, Geraedts JPM, Niermeijer MF, Staal A (1989): Anticipation in myotonic dystrophy: fact or fiction. Brain 112: 779-797

Imbert G, Kretz C, Johnson K, Mandel J-L (1993): Origin of the expansion mutation in myotonic dystrophy. Nature Genet 4: 72-76

Koch MC, Grimm T, Harley HG, Harper PS (199l) Genetic risks for children of women with myotonic dystrophy. Am J Hum Genet 48: 1084-1091

Mahadevan M, Tsilfidis C, Sabourin L, Shutler G, Amemiya C, Jansen G, Neville C, Narang M, Barceló J, O’Hoy K, Leblond S, Earle-Macdonald J, de Jong PJ, Wieringa B, Korneluk RG (1992): Myotonic dystrophy: an unstable CTG repeat in the $3^{\prime}$ untranslated region of the gene. Science 255: 1253-1255

Mahadevan MS, Amemiya C, Jansen G, Jansen G, Sabourin L, Baird S, Neville CE, Wormskamp N, Segers B, Batzer M, Lamerdin J, de Jong P, Wieringa B, Korneluk G (1993a): Structure and genomic sequence of myotonic dystrophy (DM kinase) gene. Hum Mol Genet 2: 299-304

Mahadevan MS, Foitzik MA, Surh LC, Korneluk RG (1993b): Characterization and polymerase chain reaction (PCR) detection of an Alu detection polymorphism in total linkage disequilibrium with myotonic dystrophy. Genomics 15: 446 448

Ohya K, Tachi N, Chiba S, Satoh T, Kon S, Kikuchi K, Imamura S, Yamagata H, Miki T (1994): Congenital myotonic dystrophy transmitted from an asymptomatic father with a DM-specific gene. Neurology 44: 1958-1960

Vol. 42, No. 1, 1997 
Sambrook J, Fritsch EF, Maniatis T (1989): Molecular cloning-A laboratory manual. Cold Spring Harbor Laboratory Press, New York

Shelbourne P, Winqvist R, Kunert E, Davies J, Leisti J, Thiele H, Bachmann H, Buxton J, Williamson B, Johnson K (1992): Unstable DNA may be responsible for the incomplete penetrance of the myotonic dystrophy phenotype. Hum Mol Genet 1: 467-473

Tsilfidis C, MacKenzie AE, Mettler G, Barceló J, Korneluk RG (1992): Correlation between CTG trinucleotide repeat length and frequency of severe congenital myotonic dystrophy. Nature Genet 1: 192-195

Wong LJC, Ashizawa T, Monckton DG, Caskey CT, Richard CS (1995): Somatic heterogeneity of the CTG repeat in myotonic dystrophy is age and size dependent. Am J Hum Genet $\mathbf{5 6}$ : 114-122

Yamagata H, Miki T, Ogihara T, Nakagawa M, Higuchi I, Osame M, Shelbourne P, Davies J, Johnson K (1992): Expansion of unstable DNA region in Japanese myotonic dystrophy. Lancet 339: 692

Yamagata H, Miki T, Yamanaka N, Takemoto Y, Kanda F. Takahashi K, Inui T, Kinoshita M, Nakagawa M, Higuchi I, Osame M, Ogihara T (1994): Characteristics of dynamic mutation in Japanese myotonic dystrophy. Jpn J Human Genet 39: 327-335 